\title{
Atlas statistique agricole vaudois
}

\section{Atlas statistique agricole vaudois}

Le service cantonal vaudois de l'aménagement du territoire a publié l'an dernier son cahier no 16: l'Atlas statistique agricole vaudois, par GEORGES NICOLASOBADIA. Cet ouvrage, de très grand format, comprend quelque cent cartes au 1:300000 et près de deux cents pages de textes, graphiques et tableaux. De la façon la plus approfondie, l'auteur y étudie l'évolution des structures agricoles vaudoises de 1806 à 1965.

Une telle enquête était devenue urgente tant le pays s'est transformé au cours des deux dernières générations sous l'effet de la poussée démographique et de l'industrialisation. Le besoin accru de main d'œuvre incitant les entreprises à se fixer près des villes, les campagnes se déversent vers les centres urbains; le prix des terres agricoles hausse à mesure que se rapprochent et se multiplient immeubles locatifs ou résidences secondaires. Ruiné par l'exode, face aux bouleversements à prévoir, le pays rural doit se préparer (il y va de son existence) à tenir tête et à se régénérer. Il y aura donc lieu d'élaborer une politique d'aménagement qui devra rétablir au plus vite l'équilibre entre la cité et les champs. Un tel remodelage des terres et communautés rurales n'aura de chance de réussir que si les pouvoirs publics, les services spécialisés, les équipes de recherche connaissent intimement les régions concernées, leurs problèmes humains, les données économiques et géographiques, le poids de l'histoire. Pour répondre à ces impératifs, GEORGES NICOLAS-OBADIA a composé cet ouvrage monumental, remarquable en tous points.

GEORGES NICOLAS-OBADIA a parcouru sans se lasser le pays vaudois. Attentif aux moindres détails, il s'est penché sur la multitude des statistiques communales, cantonales et fédérales, et naturellement rien ne lui a échappé des livres consacrés à la géographie et à l'histoire vaudoise.

Dans sa préface (p.12) il constate que les noms désignant les diverses régions, et qui nous viennent d'un lointain passé, ne sont pas des mots sans substance, mais des réalités quotidiennes (Lavaux, Gros de Vaud, Pays d'Enhaut). Il remarque ensuite que ces "pays» ont des limites qui varient dans le temps, certaines de leurs parties demeurant relativement stables dans l'espace. GEORGES NICOLAS-OBADIA compare ces "pays» à des fruits dont le noyau est entouré de chair; celle-ci peut selon les époques se contracter ou s'épanouir jusqu'à remplir toute la distance qui la sépare des autres fruits. "Ainsi les ,pays' agricoles vivent au rythme de l'histoire comme les fruits de l'alternance des saisons, à cette différence près que les saisons d'un ,pays' durent des siècles.» Nous rencontrons ainsi d'emblée la notion de ce qui constitue le leitmotiv de l'œuvre: le noyau régional.

Le lecteur, un peu désorienté par le foisonnement des hypothèses, des idées et des méthodes, saura gré à GEORGES NICOLAS-OBADIA d'avoir présenté en les résumant les différentes traditions intellectuelles qui s'attachèrent à définir dès le XVIe siècle les caractères originaux de l'agriculture vaudoise (p. 182). La brève étude de ces diverses manières d'aborder le sujet est passionnante et très instructive. L'auteur révèle les deux courants, historique et naturaliste (jusqu'au déterminisme), tour à tour isolés, combinés, opposés. D'une part STÜMPF (1548) et MÜNSTER (1544), RUCHAT (1714) et J. OLIVIER (1837); d'autre part RAZONMOVSKY (1789) et VUILLEMIN (1849), MARTIGNIER et CROUSAZ (1867), où la primauté des facteurs géologiques l'emporte; on retrouve enfin cette dernière conception, quoique très atténuée, chez H. GUTERSOHN (1958). A défaut de géologie, c'est le relief qui apparaît comme l'élément décisif chez H. ONDE (1971). GEORGES NICOLAS-OBADIA développe longuement la démarche originale de CH. BIERMANN $(1910,1952)$, reprise partiellement par J. FRUEH et CH. BURKY (1939 à 1945): CH. BIERMANN renoue avec les critères de différenciation spatiale (géologie, altitude, climat), puis, passant du général au particulier, il introduit quelques caractères locaux bien évidents d'origine naturelle ou dûs au travail de l'homme (forêts, vignes, dépôts glaciaires, etc). Enfin il retouche tous ces faits à l'aide de ses observations sur le terrain. Ce faisant, il distinguera par exemple le «Vrai Jorat» (altitude élevée, climat froid, humide, neigeux, forêts) et un autre Jorat dépassant de beaucoup l'espace où tous ces éléments se rencontrent simultanément. Nous voyons surgir, de façon déjà plus précise, plus scientifique les régions naturelles traditionnelles du canton de Vaud: Jorat herbager (humide et froid), Gros de Vaud (en dessous de $700 \mathrm{~m}$ ) en polyculture (terrains glaciaires variés), plaine de l'Orbe aux sols noirs (betterave). Il faut néanmoins signaler qu'en combinant ainsi dans chaque ensemble le pro-

Prof. E. L. Paillard, Société Vaudoise de Géographie, 1, Avenue Jomini, 1000 Lausanne 
duit de l'activité humaine avec un élément naturel, on n'évitera pas dans certains cas des contradictions malheureuses. Par exemple, la polyculture fourragère se pratique dans des régions aussi différentes que le Pied du Jura et l'Emmenthal, ici avec un habitat concentré et là, au contraire, dispersé. Bref tout essai de définition ou localisation régionale doit recourir à des méthodes plus éprouvées, plus exhaustives encore. Il faudra bien se reporter aux dénombrements des entreprises agricoles, quitte à procéder comme le font les géographes, pour lesquels comptent d'abord les ensembles spatiaux et leurs mouvements au cours des temps.

Les noyaux régionaux, poursuit GEORGES NICOLASOBADIA (p. 184), peuvent être repérés dans des documents de grande ancienneté déjà. Nos ancêtres avaient été frappés naturellement par des éléments marquants du paysage, tels le relief original du Vully ou les rives abruptes du Haut Léman. Dans des cas comme ceux-ci, c'était la morphologie qui avait permis de définir les limites d'une souveraineté. Une relation a pu s'établir aussi entre l'emploi d'un nom et telle production agricole; ainsi le Jorat, l'évêque de Lausanne et les droits de celui-ci sur le bois et l'herbe. Mieux encore, Lavaux (à l'origine le ravin de la Lutrive), la contribution des vignerons en produits vinicoles et la juridiction du Prieuré de Lutry et de l'évêque finissant par s'étendre sous le même nom jusqu'à la Veveyse. Aux XVle et XVIIe siècles se produit le passage du contenu spatial des régions à leurs activités agricoles. L'un et l'autre s'identifient. Désormais les noyaux vivront de leur vie propre, fluctuant dans l'espace selon que se transformaient les systèmes de culture ou les circonstances politiques, économiques du moment. Rien d'étonnant donc à ce que le Jorat herbager, forestier, à habitat dispersé ait fini au nord par perdre son individualité, là où se développait toute une région céréalière; il s'ensuivit à ses dépens l'apparition d'une nouvelle région, à prédominance céréalière: le Gros de Vaud. Voilà très résumé ce qu'on peut dire de l'approche historique des noyaux régionaux: rien de très nouveau jusqu'ici sinon l'insistance avec laquelle l'auteur souligne les fluctuations de ces régions, leur expansion ou leurs contractions. Mais d'autres méthodes vont permettre d'aborder le sujet de façon plus pénétrante encore, plus neuve et parfois plus déconcertante. C'est le recours à la statistique fouillée, au graphisme, aux mathématiques, à l'ensemble des moyens conduisant, si l'on veut aller jusqu'au bout, au mode de traitement de l'analyse quantitative. Le lecteur attentif l'aura reconnu de prime abord, en considérant la page de couverture de l'Atlas où, sur le fond vert propre au pays vaudois, se détache en noir une esquisse du canton divisé en ses régions agricoles, c'est-à-dire en une "combinaison d'éléments en interaction qui se projettent dans l'espace sous forme d'une structure spatiale ... chaque région agricole étant caractérisée par un système agricole prépondérant historiquement déterminé, en équilibre dynamique avec un certain nombre de composantes du milieu naturel».* Sur cette même page de couverture, à droite cette fois-ci, se déroule un ensemble d'ellipses élégantes qui se recouvrent, s'incluant de plus en plus étroitement jusqu'à isoler enfin le noyau régional. C'est donc la superposition de toutes ces aires réparties dans l'espace selon une stricte hiérarchie (dont l'établissement est aujourd'hui possible grâce à une inflation sans précédent des moyens d'information) qui permet d'en arriver à la connaissance précise des noyaux régionaux.

L'auteur distingue sept régions subdivisées en leurs noyaux (schéma 12 p. 60). La première, le Moyen-Pays comprend les pays agricoles de la Broye (Basse-Broye, Moudon), le Plateau (Gros de Vaud), l'Orbe et BasLéman (ouest de Nyon). Deuxième région: Bords de Lac: Vully, vignoble Nord-vaudois (Grandson), la Côte et la Petite-Côte (Pays de Morges). Troisième région: le Jorat. Quatrième région: Lavaux. Cinquième région: Chablais: Haut-Léman (Pays de Vevey-Montreux), le Pays du Rhône, les Ormonts, le Pays d'Enhaut. Sixième région: le Jura: (la Vallée, Sainte-Croix). Septième région: le Pied du Jura. L'époque de formation de ces noyaux est chaque fois indiquée. Par exemple, le Pays du Rhône: - Ier siècle au Xe siècle, et XVIe au XIXe siècles, ou encore Sainte-Croix: XVe et XVIe siècles.

On le voit: ces méthodes nouvelles de localisation ne prennent toute leur valeur que si l'on procède aux mêmes recherches à différentes époques, en recourant aux statistiques et autres données d'information échelonnées dans le passé. C'est ce qu'a fait l'auteur; c'est

*) Le système agricole se décompose en trois ordres de données et de questions: les structures sociales, la combinaison des cultures et de l'élevage à l'aide des techniques de culture. 
ce qui justifie le titre de son ouvrage: Atlas agricole vaudois de 1806 à 1965 . On verra par là que les «pays agricoles» ne sont pas des entités figées dont la situation est «étroitement déterminée par quelques faits immuables. Ce sont des réalités spatiales liées à une pratique séculaire.» Et ces réalités sont mouvantes, car elles dépendent d'éléments qui n'ont que partiellement à voir avec la nature, mais bien aussi avec l'histoire. GEORGES NICOLAS-OBADIA, sans le dire expressément, démontre que la géographie est une des disciplines les plus complexes, mais aussi les plus enrichissantes qui soient. Elle se rattache simultanément aux sciences humaines et aux sciences naturelles et mathématiques. Elle est un art, tel que l'entendaient les humanistes et nous comprenons que GEORGES NICOLAS-OBADIA ait pu dire en présentant son ouvrage qu'il était en somme un classique. De ce fait, tout en recourant pour le traitement de la statistique à l'appareil imposant des mathématiques, il ne succombe pas à certaines outrances de la nouvelle géographie quantitative.

Il n'hésitera donc pas, par exemple, à valoriser l'«événement», tenu pour négligeable par bon nombre de «quantitatifs». L'événement (c'est-à-dire une incertitude) conçu donc comme essentiel, comme l'agent d'une mutation possible; non plus une simple vague sur la surface mouvante des choses, mais l'intervention d'un homme exceptionnel, peut-être, une réforme politique ou sociale, économique ou financière, autant de faits susceptibles de modifier le cours de l'évolution.

Il nous paraît nécessaire, avant de terminer cette présentation, de résumer en peu de mots les septs commentaires si intéressants auxquels se rapportent les cartes qui constituent la seconde partie de l'ouvrage. Construits sur le même modèle, ils contiennent tous, précédant le sujet proprement dit, l'indication des sources, leur vérification, l'analyse des statistiques, les faits historiques et géographiques.

Le commentaire No 1 (pp 16 sqq) a pour sujet l'utilisation du sol et l'aire agricole dans le canton de Vaud: des calculs rigoureux aboutissant à la valeur de surface totale, de surfaces stériles, agricole, forestière et pastorale, sans omettre la surface construite ou aménagée. Relevons quelques points de cette enquête; on verra par là combien nous demeurons près des êtres et des choses. Par exemple, chaque habitant du canton a besoin en 1970 de 4.2 fois plus de surface construite ou aménagée qu'en 1806. Ou encore: à la fin du XIXe siècle, la surface stérile n'a diminué que de 550 ha (première correction des eaux du pied du Jura) (18681891). Au milieu du même siècle se produit un net réchauffement climatique. La fonte des neiges et des glaces provoque une forte diminution de la surface stérile, environ 6000 ha, ce qui permet à la longue d'étendre les pâturages, puis, par épierrement, les défrichements.

L'analyse historique, elle, relèvera la pérennité des structures spatiales agricoles vaudoises jusqu'au début de la seconde moitié du XXe siècle, où interviendra une rupture d'équilibre au profit des utilisations nonagricoles du sol. Alors commence la désagrégation de ces structures millénaires.

Le commentaire 2 (p. 41) traite des structures foncières, des types d'exploitation et de leur distribution dans l'espace vaudois (surface en propriété, faire-valoir direct, agriculteurs professionnels). On assiste à l'affaiblissement de l'emprise paysanne et vigneronne. «A partir des années 1950, nous estimons les ventes annuelles de terres cultivables (alpages non compris) aux non-paysans à 750 ha par an, soit environ la superficie agricole moyenne de deux communes vaudoises». Le commentaire 3 (pp 60 sqq), ou il est essentiellement question des noyaux régionaux, figure en résumé aux pages 116 et 117.

Dans le commentaire 4 (pp 108 sqq), GEORGES NICOLAS-OBADIA remarque que le rythme d'évolution millénaire de l'agriculture vaudoise ne sera pas véritablement ébranlé avant le XIXe siècle à son déclin: vers 1880 d'abord, lors du démarrage économique du canton et pendant l'entre deux guerres (réalisation de travaux publics pour pallier les impacts de la crise économique). Après 1945, la pression conjuguée de l'accroissement démographique, des besoins en surface par habitants, entraînés par la prospérité, va précipiter et bousculer le rythme de l'évolution (réduction massive du nombre des agriculteurs professionnels: de 15.000 à 7.000 en l'espace d'une génération). La gravité, la brusquerie de cette transformation incitent GEORGES NICOLASOBADIA à pousser plus profondément son analyse géospatiologique. Puis, dans la partie historique du commentaire, il étudie les mutations et les crises paysannes à la fin du XIXe et au début•du XXe siècle, passant ensuite aux années d'incertitude (1922-1929), à la compétition naissante entre les terres de monoculture (vignes) et la construction immobilière. Tous les sou- 
bresauts des années 1929 à 1950 (reconversion céréalière, économie de guerre, retour à la polyculture) font ressortir l'état précaire de l'agriculture vaudoise, victime des crises et, davantage encore, de l'insolente prospérité urbaine.

Le commentaire 5 (pp 136 sqq) est consacré à l'importance primordiale de l'élevage bovin. Il en relève l'effectif et l'orientation laitière très sensible à la fin du siècle dernier, après l'incorporation vaudoise dans le Marché commun helvétique. Sans relâche les paysans s'adonnent à l'amélioration de la race, un vrai renversement des rapports entre productions laitière et céréalière, à tel point que la majeure partie des marais asséchés dans les plaines du Rhône, de la Broye et de l'Orbe est transformée en prairies avant et après la Première guerre mondiale. Depuis lors, les céréales ont repris de l'importance, mais sans nuire à l'élevage, grâce à une intégration toujours plus poussée des productions végétales et animales.

Commentaire 6. Les caractéristiques d'exploitation. Commençons par un exemple qui en dit long sur les difficultés d'appréhender la statistique: il existe de grandes différences, pour les travailleurs agricoles, entre les totaux fournis par les recensements d'entreprises levés en août et en décembre de la même année. "C'est que l'introduction des «fruitières» au XIXe siècle et des machines tractées au XXe siècle a sensiblement modifié le rôle des femmes dans l'élevage et la culture des champs. De principal, le travail est devenu accessoire pour le bétail, et de permanent il n'est plus que temporaire dans la production végétale. Lorsqu'en été la rentrée des foins et l'engrangement des moissons rend leur collaboration indispensable, les chefs d'exploitation notent les femmes dans la rubrique «travailleurs» des questionnaires d'entreprises; en hiver, confinées à la maison, elles sont inscrites à la ligne "ménagères» des formules de recensement de la population (p. 165)».

Voici un autre exemple qui met en évidence la qualité du géographe sur le terrain (p. 166); à propos d'un territoire soumis à un remaniement parcellaire: «Le faux bocage sur champs avec des haies au maillage incomplet, sans chemins creux et avec des rideaux d'arbres inachevés, a disparu; il a été remplacé par des terroirs dégagés et quadrillés par un réseau serré de voies bétonnées, véritable ,centuriation' moderne». Suivent autant de remarques pertinentes à propos des systèmes agricoles de montagne, ou encore des vignes. Le commentaire 7 (dès la page 182) est l'aboutissement de cette magistrale enquête sur la régionalisation. L'auteur procède à d'ultimes vérifications. Il s'efforce de concilier les géographes modernes qui fondent leur raisonnement sur une notion synchronique du mouvement ou de la circulation et les géographes «classiques» qui réfléchissent en termes de longue durée. «Les échelles de temps ne sont pas les mêmes, dit-il, mais il existe des points de convergence, fondamentaux", que l'auteur se borne à citer brièvement pour terminer. Résumons simplement les remarques générales formulées à plus d'une reprise: les structures agricoles vaudoises d'origine millénaire ont été amenées à se réajuster. Ce pénible travail de métamorphose s'est brutalement accéléré depuis 1950. La solidité de l'ensemble ne va-t-elle pas être compromise? Cet Atlas admirable permet de connaître un témoignage à toute épreuve sur l'état de santé du pays agricole, de repérer les menaces qui se précisent, de poser le diagnostic, de proposer des mesures de salut. 